\title{
Galactosylated PLGA nanoparticles for the oral delivery of resveratrol: enhanced bioavailability and in vitro anti-inflammatory activity
}

This article was published in the following Dove Press journal: International Journal of Nanomedicine

\author{
Frederick YK Siu ${ }^{1-3}$ \\ Shaotang $\mathrm{Ye}^{1-3}$ \\ Hui Lin ${ }^{1-3}$ \\ Shoujun $\mathrm{Li}^{1-3}$ \\ 'College of Veterinary Medicine, \\ South China Agricultural University, \\ Guangzhou, People's Republic of \\ China; ${ }^{2}$ Guangdong Provincial Key \\ Laboratory of Prevention and \\ Control for Severe Clinical Animal \\ Diseases, Guangzhou, People's \\ Republic of China; ${ }^{3}$ Guangdong \\ Technological Engineering Research \\ Center for Pet, Guangzhou, People's \\ Republic of China
}

Background: Resveratrol (RES) is a natural anti-inflammatory and antioxidant compound with poor water solubility and oral bioavailability. The present study takes the advantages of nanocarriers combined with a ligand (galactose) anchoring to orally deliver RES in an attempt to improve its bioavailability and pharmacological activity.

Methods: RES-loaded galactosylated nanoparticles (RES-GNPs) were prepared by solvent diffusion technique using poly(lactic-co-glycolic acid), synthesized $N$-oleoyl-D-galactosamine and Tween 80 . RES-GNPs were characterized by particle size, morphology, entrapment efficiency (EE) and in vitro release. Oral bioavailability and in vitro anti-inflammatory activity were investigated in rats and lipopolysaccharides-induced RAW 264.7 cells, respectively.

Results: The resulting RES-GNPs were $108.4 \mathrm{~nm}$ around in particle size with a polydispersity index of 0.217. Furthermore, RES-GNPs possessed a high EE and a slow drug release in water. After oral administration, RES-GNPs significantly enhanced the oral bioavailability of RES, up to $335.7 \%$ relative to RES suspensions. In situ single-pass intestinal perfusion and cellular uptake experiments showed that GNPs could improve the intestinal permeability and transcellular transport of RES. Moreover, the anti-inflammatory efficacy of RES-GNPs in RAW 264.7 cells model was superior to free RES and RES-GNPs.

Conclusion: The results indicate that RES-GNPs can effectively promote the intestinal absorption of RES and strengthen its bioactivity, which may be a promising system for the treatment of inflammatory diseases.

Keywords: resveratrol, polymeric nanoparticles, galactosylation, oral bioavailability, antiinflammation

\section{Introduction}

Resveratrol (RES) is a natural polyphenol which is rich in a variety of plants and fruits, such as grapes, peanut and mulberry. This compound has been shown of possessing multiple pharmacological and healthy benefits, including anti-inflammation/ oxidation, ${ }^{1}$ antitumor, ${ }^{2}$ and immunoregulation. ${ }^{3}$ Currently, the French Paradox, a low incidence of cardiovascular diseases relative to a high saturated fats diet, makes this compound become popular. It is assumed to be linked with the consumption of red wine that contains a wealth of RES. However, pharmacokinetic studies revealed that the oral bioavailability of RES was extremely inadequate, ${ }^{4-6}$ due to poor solubility, chemical instability and intestinal metabolism. ${ }^{7-9}$

Encapsulation in nanoparticles (NPs) can effectively raise the intestinal supersaturated concentration, enhance the physiological stability and facilitate the transepithelial transport of poorly bioavailable molecules. ${ }^{10}$ According to the gastrointestinal
Correspondence: Shoujun Li College of Veterinary Medicine, South China Agricultural University, No 483 Wushan Road, Guangzhou 510642 , People's Republic of China Email shoujunli@scau.edu.cn 
degradation nature, NPs can be generally classified into rapidly degradable nanocarriers (eg, lipid NPs and nanoemulsions), slowly degradable nanocarriers (eg, polylactide-based NPs and polyester-based NPs) and nondegradable nanocarriers (eg, mesoporous carbons and inorganic NPs). Generally, rapidly degradable nanocarriers have great difficulty to provide a satisfactory protective effect for labile drugs in the gastrointestinal tract, whereas nondegradable nanocarriers have a poor absorption-enhancing effect. Slowly degradable nanocarriers possess unique advantages as oral delivery vehicles of poorly soluble and instable drugs, over the other two types. The degradation rate and content of slowly degradable nanocarriers, such as poly(lactic-co-glycolic acid) (PLGA) NPs, are negligible relative to their gastrointestinal transport time. Thus, such NPs can maintain a good structural integrity during the gastrointestinal transport and can provide a protection for labile drugs. Furthermore, polymeric NPs can facilitate drug transport across the intestinal epithelia through endocytosis or pinocytosis of enterocytes, ${ }^{11-13}$ thus enhancing the oral bioavailability. Nevertheless, the promotive effect of polymeric NPs on drug absorption is limited due to insufficient bioadhesion and rapid mucus turnover. ${ }^{14,15}$ If a ligand able to trigger the cytosis gets anchored, the absorptionenhancing effect of NPs will be considerably enlarged.

In the gastrointestinal tract, there are many kinds of transporters that mediate the transmembrane transport of a variety of nutrients, ${ }^{16}$ such as peptides transporters, glucose transporters and sodium-dependent vitamins cotransporters. Among all sorts of monosaccharides, galactose exhibits the fastest absorption rate in the intestine, which is mediated by sodium glucose-linked transporter 1 (SGLT1), an intestinal $\mathrm{Na}^{+}$-glucose cotransporter. ${ }^{17}$ It is indicative that the galactose moiety may provide compounds with an improved route of intestinal transport by the way of SGLT1. ${ }^{18}$ Recently, nanostructured lipid carriers (NLCs) have been employed for RES encapsulation. ${ }^{19}$ The constructed NLCs based on PEG-40 stearate demonstrated enhanced tyrosinase inhibitory activity. Pujara et al developed RES-encapsulated soy protein isolate nanocomplexes and tested the performance for solubility and dissolution improvement. ${ }^{20}$ Mesoporous silica was also utilized to encapsulate RES in an attempt to ameliorate its dissolution, permeability and anti-inflammatory response..$^{21}$ These delivery systems, to some extent, improved the physicochemical properties and pharmacological activities of RES. Nonetheless, a more efficient and smart drug delivery system is still desired to potentiate the potency of RES.

In this study, we developed a galactosylated PLGA NPs (GNPs) to orally deliver RES, aiming to enhance the oral bioavailability and in vitro anti-inflammatory effect. Galactosylation was achieved through surface engineering of NPs by introducing $N$-oleoyl-D-galactosamine into the formulation. RES-loaded GNPs (RES-GNPs) were prepared by solvent diffusion method and characterized by particle size, entrapment efficiency (EE) and in vitro release. The formulation performances of RES-GNPs were evaluated with in situ single-pass intestinal perfusion, cellular uptake, in vivo pharmacokinetics and in vitro anti-inflammation in lipopolysaccharide (LPS)-induced RAW264.7 cells.

\section{Materials and methods Materials}

RES (transisomer) was purchased from Shaanxi Huike Botanical Development Co., Ltd. (Xi'an, People's Republic of China). PLGA (DL-lactide:glycolide $=75: 25$, $\mathrm{Mw}=\sim 10,000$ ) was obtained from RESENBio Technology Co., Ltd (Xi'an, People's Republic of China). Galactosamine hydrochloride, galactose, LPS, chlorpromazine and simvastatin were purchased from Sigma-Aldrich (St Louis, MO, USA). Oleoyl chloride, triethylamine and Tween 80 were provided by Sinopharm Chemical Reagent (Shanghai Shi, People's Republic of China). Filipin and 3-amino-6-chloro$\mathrm{N}$-(diaminomethylene)-5-(ethyl(isopropyl)amino)pyrazine2-carboxamide (EIPA) were purchased from APExBIO (Shanghai, China). Purified water was produced by a Milli-Q water purifier (Merck Millipore, MA, USA). All other chemicals were of analytical grade and used as provided.

\section{Synthesis of $\mathrm{N}$-oleoyl-D-galactosamine}

The synthesis of amphiphilic $N$-oleoyl-D-galactosamine was based on galactosamine hydrochloride and oleoyl chloride in the presence of triethylamine. Briefly, galactosamine hydrochloride was dissolved in deionized water followed by addition of triethylamine. Then, a slight excess of oleoyl chloride was slowly added to start the acylation reaction. The reaction was maintained for $24 \mathrm{~h}$ at room temperature. The crude products were precipitated against acetone and then washed with methanol several times. The purified product was characterized by ${ }^{1} \mathrm{HNMR}$ using deuterated dimethyl sulfoxide $\left(\mathrm{DMSO}_{\mathrm{f}}\right)$ as a solvent.

\section{Preparation of RES-GNPs}

RES-GNPs were prepared by a solvent diffusion method. ${ }^{22}$ Briefly, RES and PLGA were dissolved in acetone to form an organic phase; $N$-oleoyl-D-galactosamine and Tween 80 were dissolved in purified water. Then, the organic phase was rapidly injected into the aqueous phase under stirring 
at 8,000 rpm. Upon acetone diffusion into the water, the materials self-assembled into RES-GNPs. Afterward, the residual solvent was removed under reduced pressure using a rotatory evaporator. The factors influencing the formulation properties were screened by changing one variable and keeping others unchanged, including the amount of PLGA, the amount of $N$-oleoyl-D-galactosamine and the concentration of Tween 80 in the system.

\section{Characterization of RES-GNPs}

The particle size and $\zeta$ potential of RES-GNPs were measured at $25^{\circ} \mathrm{C}$ based on the dynamic light scattering and electrophoretic mobility principles using Zetasizer Nano ZS analyzer (Malvern Instruments Ltd, Malvern, UK). The samples were diluted 25 times with purified water before determination. After equilibrating for $120 \mathrm{~s}$, the samples were subjected to laser diffraction or Doppler velocimetry for the output of particles size and $\zeta$ potential. The morphology of RES-GNPs was inspected by transmission electron microscope (TEM) (Tecnai 10; Philips, Amsterdam, the Netherlands).

The EE of RES-GNPs was determined by the centrifugal ultrafiltration technique with a minor modification. ${ }^{23}$ The unentrapped RES was first removed by centrifugation at 15,000 rpm for $5 \mathrm{~min}$. Then, the upper layer of RESGNPs was pipetted and placed in a centrifugal filter device (Amicon ${ }^{\circledR}$ Ultra-0.5, MWCO 5,000; Merck Millipore). Afterward, the procedure of centrifugal ultrafiltration was performed to separate free RES from the system. The concentration of free RES $\left(C_{\text {fre }}\right)$ in the filtrate was determined by high performance liquid chromatography (HPLC) at $306 \mathrm{~nm}$ (Dionex Ultimate 3000 HPLC; Acclaim ${ }^{\mathrm{TM}} 120 \mathrm{C}_{18}$ column ( $5 \mu \mathrm{m}, 4.6 \times 150 \mathrm{~mm})$; Thermo Fisher Scientific, MA, USA). The mobile phase consisted of $30 \%$ acetonitrile and $70 \%$ phosphoric acid solution $(0.05 \%, \mathrm{v} / \mathrm{v})$ pumped at a flow rate of $1.0 \mathrm{~mL} / \mathrm{min}$. EE was calculated by the following equation: $\mathrm{EE}(\%)=\left(1-C_{\text {fre }} / C_{\text {tot }}\right) \times 100 \%$, where $C_{\text {tot }}$ denoted the total concentration of RES in the system.

\section{In vitro release study}

The in vitro release of RES-GNPs was studied using the dialysis bag method with RES solution and RES-loaded NPs (RES-NPs) as references. RES solution was prepared by dissolving RES in the cosolvent of water/ethanol/ PEG400 (20/40/40, v/v/v). RES-NPs were prepared following the same procedure of RES-GNPs. Aliquots of samples (equivalent to $10 \mathrm{mg}$ of RES) were placed into the dialysis bags (MWCO 14,000) and sealed. Then, the bags loading RES preparations were dialyzed against $100 \mathrm{~mL}$ of purified water at $37^{\circ} \mathrm{C}$, in which $1 \%$ Tween 80 added as the solubilizing agent. At predetermined time points, $200 \mu \mathrm{L}$ of release medium were withdrawn and immediately replenished with the same volume of fresh medium. The release medium was then diluted with $200 \mu \mathrm{L}$ of methanol and subjected to HPLC analysis for RES quantification. The release curves were plotted based on the accumulative release percentage vs time.

\section{Oral bioavailability study}

The animal experiments were conducted with the Guidelines on the Care and Use of Animals for Scientific Purposes (2004). The experimental protocols were reviewed and approved by the Experimental Animal Welfare Ethics Committee of South China Agricultural University. Sprague-Dawley (SD) rats $(220 \pm 20 \mathrm{~g})$ were fasted overnight before administration but freely accessible to water. The rats were randomly divided into four groups $(n=5)$, ie, RES suspensions (dispersed in 0.3\% CMC-Na solution) (introgastric [ig]), RESNPs (ig), RES-GNPs (ig) and RES solution (intravenous). Rats were given RES preparations by gavage $(40 \mathrm{mg} / \mathrm{kg}$ ) or intravenous injection $(8 \mathrm{mg} / \mathrm{kg})$. Approximately $0.25 \mathrm{~mL}$ of blood was collected into the heparinized tube from the tail vein of rats at $0.133,0.25,0.5,1,2,3,5,8$ and $12 \mathrm{~h}$ following administration. The blood samples were subsequently centrifuged at 4,500 rpm for $5 \mathrm{~min}$ to prepare the plasma.

To quantify the plasma RES, a liquid-liquid extraction procedure was used to retrieve RES from the plasma. In brief, $100 \mu \mathrm{L}$ of plasma was mingled with $500 \mu \mathrm{L}$ of acetonitrile. After vortex for $5 \mathrm{~min}$, the mixtures were centrifuged at $13,000 \mathrm{~g}$ for $15 \mathrm{~min}$. The supernatants were transferred to new tubes followed by evaporation at $30^{\circ} \mathrm{C}$ under vacuum using a concentrator (Eppendorf, NY, USA). The residues were reconstituted in $150 \mu \mathrm{L}$ of $50 \%$ acetonitrile for UPLC-QTOF/MS analysis (Xevo G2 QTOF, Waters). Elution was performed using a gradient procedure at a flow rate of $0.45 \mathrm{~mL} / \mathrm{min}$ using $0.1 \%$ formic acid in water (mobile phase $\mathrm{A}$ ) and $0.1 \%$ formic acid in acetonitrile (mobile phase B). The gradient elution was $10 \% \mathrm{~B}$ at $0-1 \mathrm{~min}, 10 \%-95 \% \mathrm{~B}$ at $1-3 \mathrm{~min}$, $95 \% \mathrm{~B}$ at $3-4.5 \mathrm{~min}$ and $95 \%-10 \% \mathrm{~B}$ at $4.5-5 \mathrm{~min}$. The plasma RES was quantified based on extracted ion chromatograms using MassLynx. PKSolver 2.0, a freely available add-in Excel program, was used to process the data and extract the pharmacokinetic parameters.

\section{In situ single-pass intestinal perfusion}

To appreciate the effect of unmodified NPs and GNPs on the intestinal permeability of RES, in situ single-pass intestinal perfusion was carried out following the reported 
procedure. ${ }^{23,24} \mathrm{SD}$ rats were fasted overnight prior to the experiment but allowed free access to water. The rats were anesthetized with $20 \%$ urethane $(1.0 \mathrm{~g} / \mathrm{kg}$ ) by intraperitoneal injection. The intestine was exposed by making an incision in the midline of the abdomen. The proximal and distal ends of duodenum, jejunum, ileum and colon were identified and cannulated with silicone tubes $(\Phi 2.5 \times 4 \mathrm{~mm})$. A pH 7.4 Krebs Ringer's buffer (KRB) was used to rinse the intestinal contents. Perfusion solutions were prepared by diluting RES solution, RES-NPs or RES-GNPs with KRB to $25 \mu \mathrm{g} / \mathrm{mL}$. After preperfusion for $30 \mathrm{~min}$, the perfusates were collected per $15 \mathrm{~min}$ up to $120 \mathrm{~min}$. Finally, the diameter and length of the intestinal segments were measured. The net water flux in the perfusion experiment was calibrated by the gravimetric method based on the sham-operated group. The effective permeability coefficient $\left(P_{\text {eff }}\right)$ was calculated according to the inlet and outlet concentrations of RES using the following equation:

$$
P_{\text {eff }}=-\frac{Q}{2 \pi r L} \ln \frac{C_{\text {out }}}{C_{\text {in }}}
$$

where $Q$ denotes the flow rate $(0.2 \mathrm{~mL} / \mathrm{min}), r$ and $L$ represent the radius and length of the perfused intestinal segment (cm), $C_{\text {in }}$ and $C_{\text {out }}$ are the inlet and outlet concentrations of RES, respectively.

\section{Cellular uptake and internalization}

Caco-2 cells from American Type Culture Collection were cultured in Dulbecco's Modified Eagle's Medium (DMEM) at $37^{\circ} \mathrm{C}$ under $5 \% \mathrm{CO}_{2}$ atmosphere, in which fetal bovine serum (FBS, $10 \%$ ) and penicillin-streptomycin solution $(100 \mathrm{IU} / \mathrm{mL})$ were supplemented. For cellular uptake study, Caco-2 cells were seeded in 12-well plate at a density of $5 \times 10^{4}$ cells/well and cultured for $24 \mathrm{~h}$. After that, the culture medium was removed and replaced with DMEM-diluted RES, RES-NPs or RES-GNPs $(20 \mu \mathrm{g} / \mathrm{mL})$. The cells were treated for 1,2 and $4 \mathrm{~h}$ at $37^{\circ} \mathrm{C}$, respectively, then washed twice with cold PBS (pH 7.4) and followed by lysis with $60 \%$ acetonitrile. After vortex for $2 \mathrm{~min}$, the lysates were centrifuged at 13,000 rpm for 5 min under $4^{\circ} \mathrm{C}$ to obtain the supernatant. RES in the supernatant was then quantified by HPLC.

To ascertain the cellular trafficking mechanism of RESGNPs, the cellular uptake of RES-GNPs was analyzed in the presence of various transport inhibitors or competitors. ${ }^{25}$ Caco-2 cells were pretreated with hypertonic sucrose $(0.4 \mathrm{M})$, chlorpromazine $(20 \mu \mathrm{M})$, simvastatin $(50 \mu \mathrm{M})$, Filipin $(1.5 \mu \mathrm{M})$, EIPA $(20 \mu \mathrm{M})$ or galactose $(50 \mu \mathrm{M})$ at $37^{\circ} \mathrm{C}$ for $0.5 \mathrm{~h}$. Then, RES-GNPs $(20 \mu \mathrm{g} / \mathrm{mL})$ were added and continued to incubate for $2 \mathrm{~h}$. Another batch of cells were used to investigate the cellular uptake of RES-GNPs under $4^{\circ} \mathrm{C}$. Thereafter, the cells were washed, lysed and centrifuged at $13,000 \mathrm{rpm}$. RES concentration in the supernatants was determined by HPLC. The cellular trafficking mechanism of RESGNPs was interpreted based on the changes in cellular uptake.

To investigate the cellular internalization of RES-GNPs, Caco- 2 cells were cultured in a 6-well plate containing round microslides for $24 \mathrm{~h}$. Subsequently, the cells were incubated with coumarin 6-labeled RES-NPs and RES-GNPs at $37^{\circ} \mathrm{C}$ for $30 \mathrm{~min}$. After that, the cells were washed twice with cold PBS and fixed with $4 \%$ paraformaldehyde for $30 \mathrm{~min}$. The internalized RES-GNPs was visualized on Caco-2 cells by confocal laser scanning microscopy after nucleus staining with DAPI.

\section{In vitro anti-inflammatory effect}

The anti-inflammatory effect of RES-GNPs was evaluated in vitro with LPS-induced inflammatory model. ${ }^{26}$ Mouse macrophage cell line RAW 264.7 was purchased from the Type Culture Collection of Chinese Academy of Sciences (Beijing, People's Republic of China). Cells were cultured in RPMI-1640 medium supplemented with 10\% FBS (Thermo Fisher Scientific) at $37^{\circ} \mathrm{C}$ in $5 \% \mathrm{CO}_{2}$. RAW 264.7 cells were seeded in 24-well plates at $2 \times 10^{5}$ cells/well and then treated with LPS $(1 \mu \mathrm{g} / \mathrm{mL})$ in the absence or presence of tested samples at indicated concentration for $24 \mathrm{~h}$. The cells treated with culture medium and LPS alone were designated as the control and model group, respectively. The supernatants of cells were isolated by centrifugation at 10,000 rpm under $4^{\circ} \mathrm{C}$ after incubation with RES solution, RES-NPs and RES-GNPs. The inflammatory mediators of tumor necrosis factor $\alpha$ (TNF- $\alpha$ ), interleukin-6 (IL-6) and nitric oxide (NO) were measured using their ELISA kits (ExCell Bio, Shanghai, China), according to the manufacturers' instructions.

\section{Results \\ Synthesis and identification of $\mathrm{N}$-oleoyl- D-galactosamine}

The synthesis of $N$-oleoyl-D-galactosamine was accomplished via the acylation between galactosamine and oleoyl chloride. The resultant product exhibited a distinct thin layer chromatographic signal from the reactants (data not shown). ${ }^{1} \mathrm{HNMR}$ was utilized to verify the chemical structure of the product. Figure 1 shows the ${ }^{1} \mathrm{HNMR}$ spectrum of the product. It clearly displayed the proton signals of galactosamine $(\delta 3.42$, $3.64,3.85$ and 7.47) and oleoyl ( $\delta 1.25,1.49,2.18$ and 5.44), indicating successful synthesis of $N$-oleoyl-D-galactosamine. The galactosamine moiety of $N$-oleoyl-D-galactosamine 


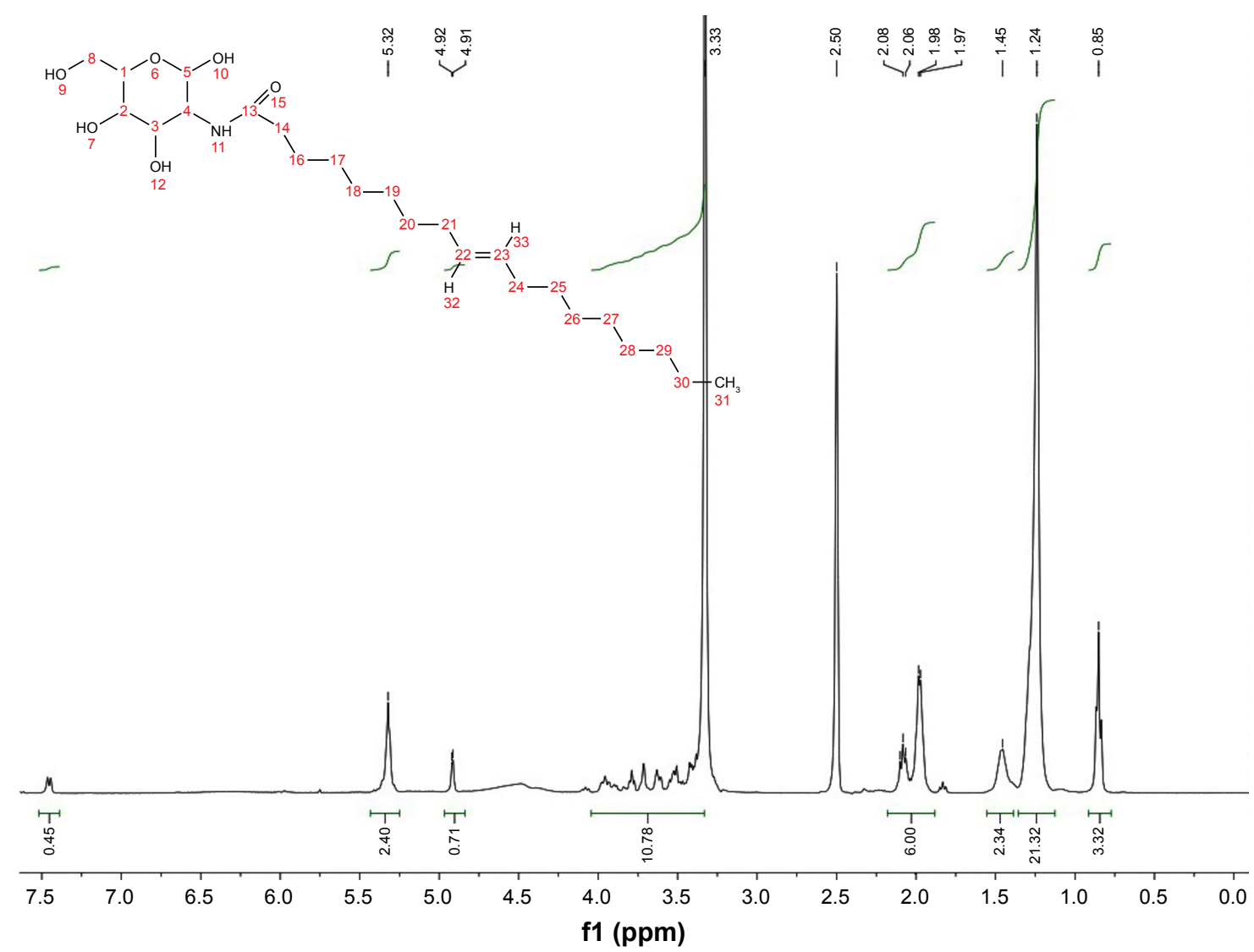

Figure I Synthesis and 'HNMR identification of N-oleoyl-D-galactosamine.

possesses a hydrophilic property, whereas the oleoyl moiety is hydrophobic. This amphiphilic nature imparts this compound an excellent surface activity that can be used to modify NPs and facilitate drug loading.

\section{Preparation and characterization of RES-GNPs}

The solvent diffusion method is a facile and straightforward preparative process of polymeric NPs. ${ }^{27,28}$ The formulation constitutions influence the formulation performance, such as particle size, drug loading and in vivo transport. The effects of formulation variables on the particle size and EE of RES-GNPs are shown in Figure 2. The amount of PLGA in the formulation greatly affected the particle size of RES-GNPs, other than EE. The formulation containing a higher level of PLGA resulted in larger NPs. It indicates that less PLGA tends to form smaller RES-GNPs when using a fixed amount of surfactant. $N$-oleoyl-D-galactosamine had
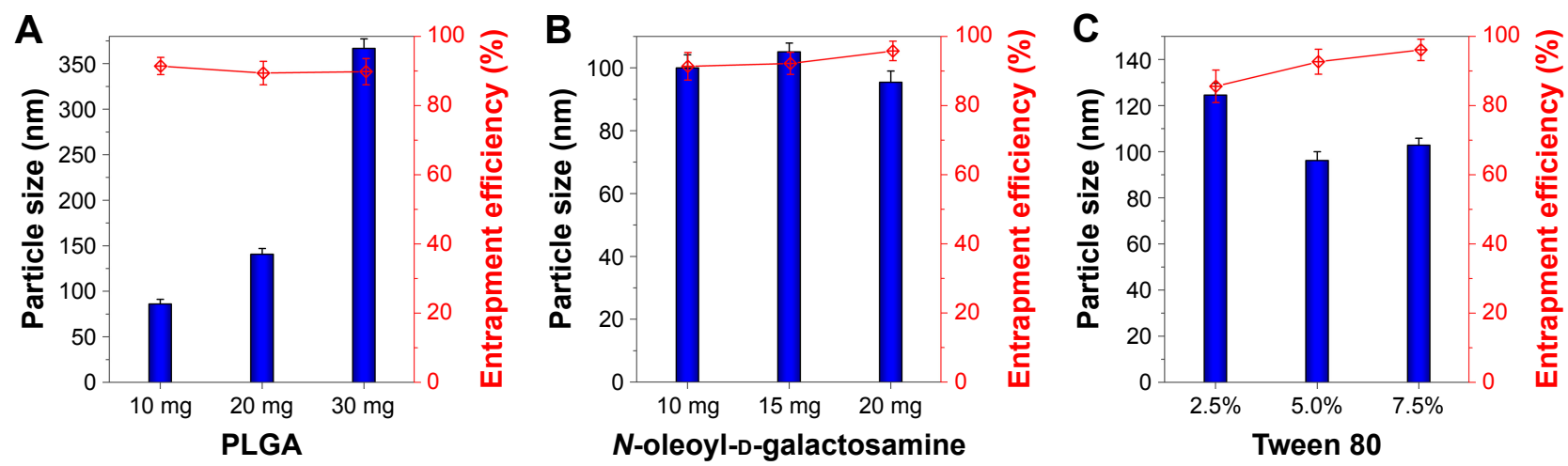

Figure 2 Factors influencing the formulation properties (particle size and entrapment efficiency) of RES-GNPs: PLGA vs 10 mg of RES, 10 mg of N-oleoyl-D-galactosamine and I $25 \mu \mathrm{L}$ of Tween 80 (A); N-oleoyl-D-galactosamine vs $10 \mathrm{mg}$ of RES, $10 \mathrm{mg}$ of PLGA and I25 $\mu \mathrm{L}$ of Tween 80 (B); and Tween 80 vs 10 mg of RES, 10 mg of PLGA and $20 \mathrm{mg}$ of $\mathrm{N}$-oleoyl-D-galactosamine (C).

Abbreviations: PLGA, poly(lactic-co-glycolic acid); RES, resveratrol; RES-GNPs, RES-loaded galactosylated nanoparticles. 
a certain effect on the particle size and EE of RES-GNPs. High ratio of $N$-oleoyl-D-galactosamine was favorable to reduce the particle size and increase the EE, which may be associated with the surface activity of this compound that reduced the interfacial tension by inserting the interfacial membrane of NPs. On the other hand, instantaneous and prompt formation of NPs was conducive to enabling drug entrapment timely. The effect of Tween 80 on the formulation performances was also significant where high level of Tween 80 resulted in smaller particle size of RES-GNPs. However, there was no difference between 5\% level and $7.5 \%$ level, indicating that Tween 80 positively facilitated the formation of RES-GNPs under a certain level. Similar to $N$-oleoyl-D-galactosamine, Tween 80 lent a hand with the entrapment of RES as a surfactant.

Considering the advantages of small particle size and high EE in drug delivery, the final formulation was typically determined as $10 \mathrm{mg}$ of RES, $10 \mathrm{mg}$ of PLGA, $20 \mathrm{mg}$ of $N$-oleoyl-D-galactosamine and $125 \mu \mathrm{L}$ of Tween 80 formulated into $2.5 \mathrm{~mL}$ of water. The resulting RES-GNPs were $108.4 \mathrm{~nm}$ in particle size with a polydispersity index of 0.217 (Figure 3A). RES-GNPs appeared a spherical morphology as
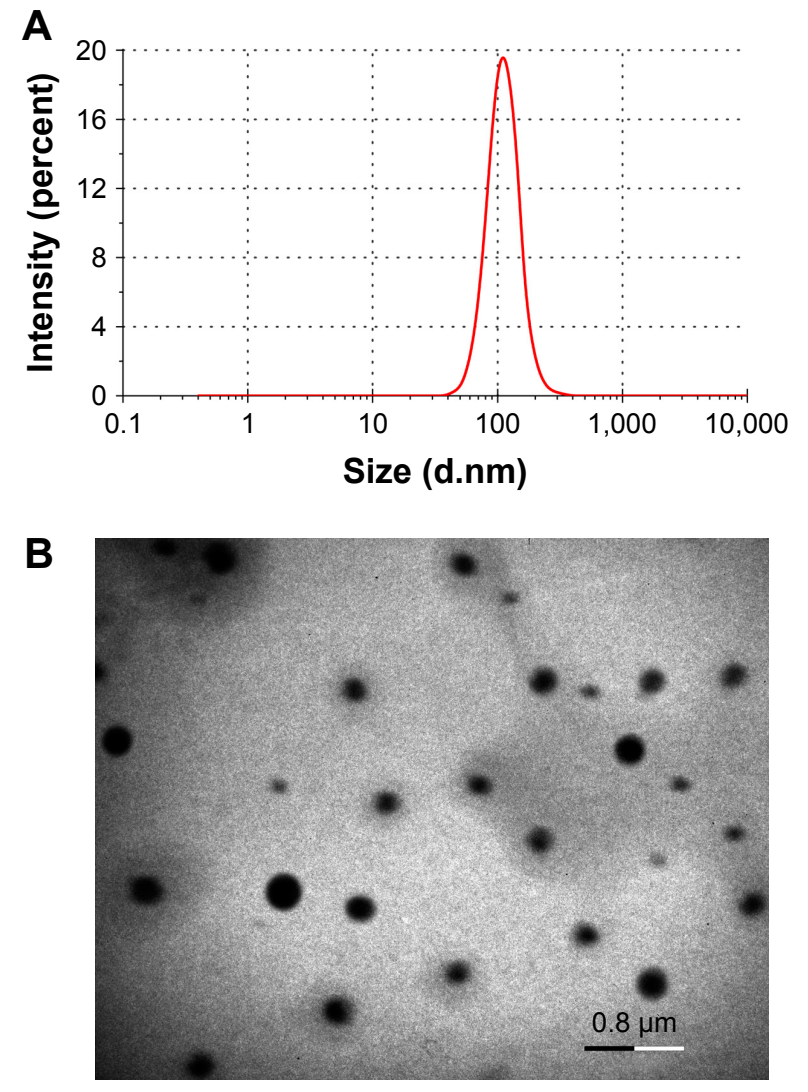

Figure 3 Particle size distribution (A) and TEM micrographs of RES-GNPs (B). Abbreviations: TEM, transmission electron microscopy; RES, resveratrol; GNPs, galactosylated nanoparticles. observed by TEM (Figure 3B). The particle size as gauged by the scale bar was $\sim 150 \mathrm{~nm}$, which was a little larger than the hydrodynamic size measured by dynamic light scattering, which may be associated with the flattening of particles upon sampling. The EE of RES-GNPs was determined to be $97.22 \% \pm 2.31 \%$, showing an excellent drug entrapment capacity. Of note, RES-GNPs was negatively charged with a $\zeta$ potential of $-46.3 \mathrm{mV}$. The absolute $\zeta$ potential $>25 \mathrm{mV}$ also suggests a high colloidal stability of RES-GNPs due to the electrostatic repulsion. ${ }^{29}$ The physical stability of RESGNPs was shown to be fine for a short-term storage survey. The significant changes of particle size and polydispersity index of nanoparticles did not occur.

\section{In vitro drug release}

The release profiles of RES from RES-NPs and RES-GNPs are shown in Figure 4. Free RES (solution formulation) was released completely in a very short time, indicating less release resistance providing with the dialysis bag. Both RES-NPs and RES-GNPs exhibited a slow release process, where the accumulative release percentages of two formulations were less than $43 \%$ within $8 \mathrm{~h}$. The result may suggest that slow release allows the majority of drug to be retained in NPs when transporting through the gastrointestinal tract, which is favorable for RES absorption via NPs. It still had $20 \%$ of drug that has not been released at the time point of $36 \mathrm{~h}$. However, there was no difference in release between RES-NPs and RES-GNPs. This may be ascribed to the similar matrix structure between RES-NPs and RES-GNPs, in which RES shares an identical crystallinity property. The participation of $\mathrm{N}$-oleoyl-D-galactosamine has less impact

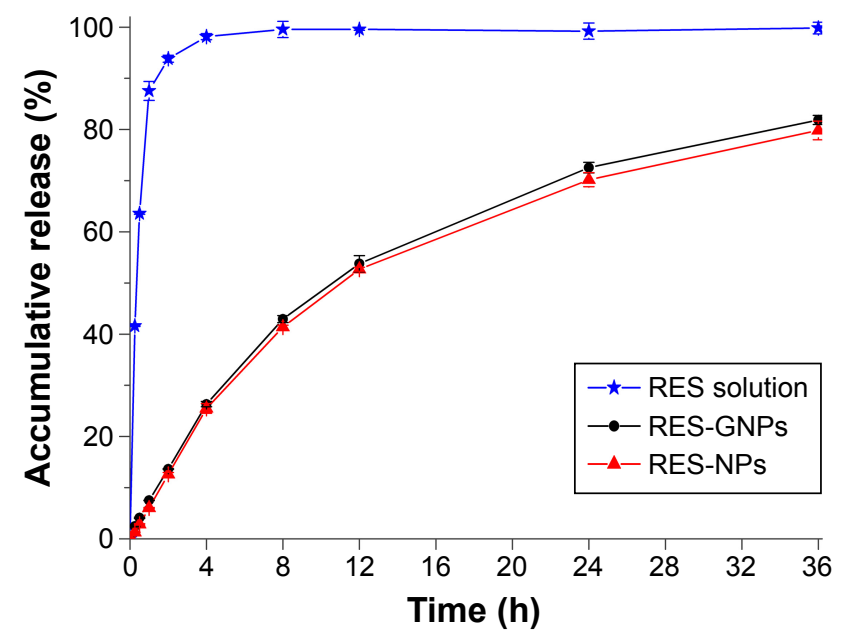

Figure 4 Release curves of RES from RES-NPs and RES-GNPs performed in water based on the dialysis bag method.

Note: Data expressed as mean $\pm S D(n=3)$.

Abbreviations: RES, resveratrol; RES-GNPs, RES-loaded galactosylated nanoparticles; RES-NPs, RES-loaded nanoparticles. 


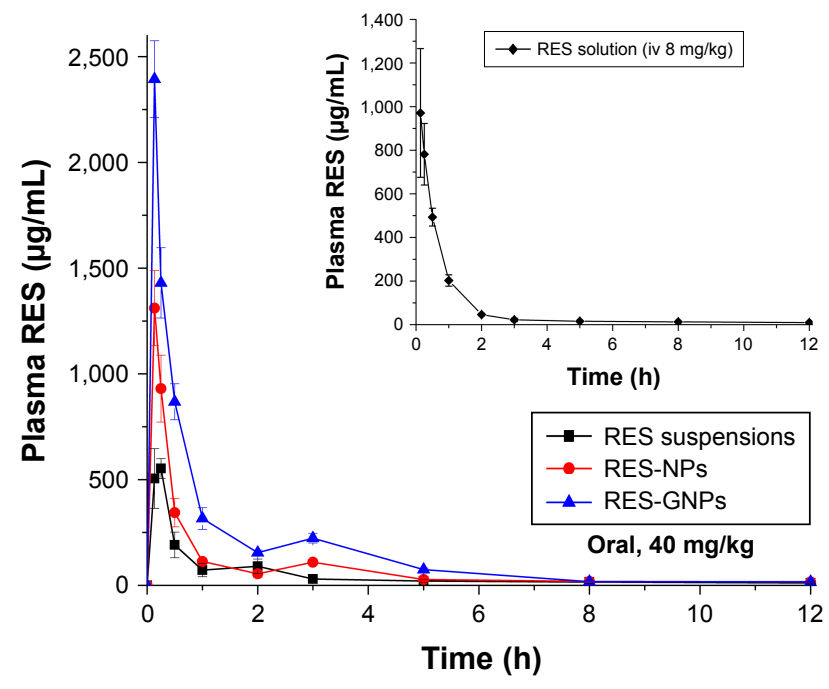

Figure 5 Pharmacokinetic profiles of plasma RES concentration vs time after ora administration of RES suspensions, RES-NPs and RES-GNPs at a dose of $40 \mathrm{mg} / \mathrm{kg}(\mathrm{n}=5)$. Note: The inset represents the pharmacokinetics of RES solution dosed by intravenous injection $(8 \mathrm{mg} / \mathrm{kg})$.

Abbreviations: RES, resveratrol; RES-NPs, RES-loaded nanoparticles; RES-GNPs, RES-loaded galactosylated nanoparticles.

on the crystal matrix of nanoparticles. In order to elucidate the release mechanism, the release curves were fitted with various models. It shows that the in vitro releases of RES-NPs and RES-GNPs conform to the first-order kinetic model $\left(R^{2}=0.9948\right)$, which belongs to the diffusion-based release process.

\section{Enhanced bioavailability}

The profiles of plasma RES concentration vs time in rats are shown in Figure 5. The main pharmacokinetic parameters extracted from noncompartment model are listed in Table 1 . The blood drug level sharply declined in the case of RES solution (inset), indicating that RES can quickly distribute and be eliminated in vivo once injected into the blood vessel. For three formulations, they all exhibited a rapid absorption after oral administration. The maximum plasma concentration $\left(C_{\max }\right)$ appeared at the first time point, showing that the intestinal absorption of RES is not as poor as expected, although limited. RES-NPs brought about a quicker and higher degree of RES absorption compared with RES suspensions. Nevertheless, RES-GNPs resulted in the best absorption of RES after oral administration. The $C_{\max }$ of RES-GNPs was almost two times as high as that of RESNPs. These results indicate that NPs can positively improve the oral absorption of RES, though GNPs can strengthen the absorption-enhancing effect. Of note, the blood drug concentration vs time curves presented a double peak for all RES formulations, suggesting that an enterohepatic circulation may take place on RES. ${ }^{30}$ The relative bioavailability of RES-NPs was $165.7 \%$ compared with RES suspensions, whereas RES-GNPs yielded a higher bioavailability, up to $335.7 \%$. RES-GNPs significantly enhanced the oral bioavailability of RES perhaps due to galactosylation.

In general, gastrointestinal absorption of substances can be carried through the passive, carrier-mediated and cytosis (membrane mobile) transport. ${ }^{31}$ For small molecular compounds, passive- and carrier-mediated transports play the basic role in their gastrointestinal absorption. In terms of macromolecules or NPs, cytosis transport is the principal mechanism of transepithelial absorption. The intestinal absorption of galactose is fulfilled by way of SGLT1, ${ }^{32}$ a transporter-mediated process. Surface modification with galactose moiety imparts NPs a high affinity and specificity for enterocytes, which can facilitate NPs transport across the absorptive epithelia by cytosis or carrier-mediated transport. Zhang et al developed galactosylated chitosan-based NPs for the oral delivery of a mitogen-activated protein kinase 4 (Map4k4) siRNA, finding that GNPs more efficiently promoted siRNA distribution into the ulcerative colon following oral administration. ${ }^{33}$ Jain et al also demonstrated that galactosylated solid lipid NPs could significantly enhance the cellular uptake of doxorubicin. ${ }^{34}$ Our findings accorded with the reported outcomes, showing that galactosylation may be a promising strategy to ameliorate the oral delivery of NPs, hence the bioavailability.

Table I Main pharmacokinetic parameters of RES after oral administration of RES suspensions, RES-NPs and RES-GNPs or intravenous injection of RES solution in rats $(n=5)$

\begin{tabular}{|c|c|c|c|c|}
\hline Formulations & RES solution (iv) & RES suspensions & RES-NPs & RES-GNPs \\
\hline$C_{\max }(\mathrm{ng} / \mathrm{mL})$ & $974.3 \pm 302.5$ & $553 \pm 54.1$ & $I, 31 I \pm 189$ & $2,393 \pm 182 * *$ \\
\hline$T_{\max }(\mathrm{h})$ & $0.133 \pm 0.054$ & $0.252 \pm 0.052$ & $0.137 \pm 0.057$ & $0.135 \pm 0.052$ \\
\hline $\mathrm{AUC}_{0-\mathrm{t}}(\mathrm{ng} / \mathrm{mL} * \mathrm{~h})$ & $902.5 \pm 33.7$ & $564.1 \pm 8 I .3$ & $934.9 \pm|| 2.4$ & $1,894 \pm 150.7 * *$ \\
\hline $\mathrm{MRT}_{0-\infty}(\mathrm{h})$ & $5.274 \pm 0.931$ & $6.903 \pm 1.032$ & $6.912 \pm 0.941$ & $2.333 \pm 1.511 * *$ \\
\hline Absolute BA (\%) & l & $12.50 \pm 1.796$ & $20.72 \pm 8.99$ & $41.97 \pm 3.34 * *$ \\
\hline Relative BA (\%) & 1 & I & $165.7 \pm 19.92$ & $335.7 \pm 26.72 * *$ \\
\hline
\end{tabular}

Notes: BA: bioavailability calculated based on AUC ${ }_{0-t}$; ANOVA, $* * P<0.01$, compared with RES-NPs.

Abbreviations: RES, resveratrol; RES-NPs, RES-loaded nanoparticles; RES-GNPs, RES-loaded galactosylated nanoparticles; AUC, area under curve; MRT, mean retention time; BA, bioavailability; iv, intravenous. 
Table 2 The effective intestinal permeability $\left(P_{\text {eff }}\right)$ of free RES, RES-NPs and RES-GNPs determined by in situ single-pass intestinal perfusion $(n=3)$

\begin{tabular}{llll}
\hline $\begin{array}{l}\text { Intestinal } \\
\text { segment }\end{array}$ & $\boldsymbol{P}_{\text {eff }}$ & & RES-NPs \\
\cline { 2 - 4 } & Free RES & $5.138 \pm 0.366 \times 10^{-4, \ldots}$ & $8.290 \pm 0.349 \times 10^{-4, * *}$ \\
\hline Duodenum & $1.622 \pm 0.169 \times 10^{-4}$ & $7.038 \pm 0.599 \times 10^{-4}$ & $1.072 \pm 0.293 \times 10^{-3, *}$ \\
Jejunum & $5.662 \pm 1.262 \times 10^{-4}$ & $9.082 \pm 0.132 \times 10^{-4, \#}$ & $1.703 \pm 0.502 \times 10^{-3, * *}$ \\
Ileum & $7.100 \pm 0.826 \times 10^{-4}$ & $3.212 \pm 0.432 \times 10^{-4}$ & $1.794 \pm 0.108 \times 10^{-3, * *}$ \\
Colon & $3.112 \pm 0.632 \times 10^{-4}$ &
\end{tabular}

Notes: Paired $t$-test, ${ }^{\# P} P 0.01,{ }^{\#} P<0.05$, compared with free RES; $* * P<0.01, * P<0.05$, compared with RES-NPs.

Abbreviations: RES, resveratrol; RES-NPs, RES-loaded nanoparticles; RES-GNPs, RES-loaded galactosylated nanoparticles.

\section{Intestinal permeability}

The $P_{\text {eff }}$ of free RES, RES-NPs and RES-GNPs is listed in Table 2. Free RES exhibited a moderate intestinal permeability, up to the exponential order of $10^{-4}$. Aside from the duodenum and colon, the limited step for RES absorption should be its intrinsic solubility and metabolism, ${ }^{35}$ rather than the permeability. Nevertheless, the intestinal permeability of RES was also significantly improved when encapsulated into NPs, especially for the sites of duodenum and ileum. The improvement in permeability was more noticeable in the case of RES-GNPs. RES-GNPs could enhance the permeability of RES in all investigated intestinal segments compared with RES-NPs. We assume that the permeability optimization can be attributed to galactose attachment of NPs that mediates and facilitates the internalization of NPs into the enterocytes. Galactose-modified NPs have great advantages in heightening the drug's permeability and absorptivity by virtue of excellent intestinal affinity.

\section{Cellular uptake and internalization}

Figure 6 presents the cellular uptake of free RES, RES-NPs and RES-GNPs in Caco-2 cells. The solution formulation (free RES) exhibited a rapid cellular uptake relative to RES-NPs at 1, 2 and $4 \mathrm{~h}$. It may be attributive to the small molecular dimension of RES and effortless approachability to the cells via the transcellular transport. The cellular uptake of RES-NPs can be conducted by the membrane mobile transport, thus the transport efficiency is comparatively slow. However, there was a significant difference in cellular uptake between RES-GNPs and RES-NPs. The cellular uptake level of RES-GNPs was parallel to that of free RES at 1 and $2 \mathrm{~h}$, but significantly higher than RES-NPs. It was noted that the cellular uptake of RES-GNPs incessantly increased over time, which was also different from RES solution. At the time point of $4 \mathrm{~h}$, the cellular uptake of RES-GNPs was larger than that of RES solution. These results demonstrate that there is a different mechanism responsible for the cellular uptake of RES-GNPs.
To clarify the cellular trafficking mechanism of RESGNPs, we investigated the cellular uptake of RES-GNPs in the presence of different physiological inhibitors, competitor (galactose) or under a lower temperature $\left(4^{\circ} \mathrm{C}\right)$. Figure 7 shows the relative cellular uptake of RES-GNPs in limited conditions. The cellular uptake of RES-GNPs was significantly inhibited by chlorpromazine and galactose, whereas hypertonic sucrose, simvastatin, Filipin and EIPA almost had no effect on the cellular uptake. Chlorpromazine, a specific inhibitors of clathrin-mediated endocytosis, reduced the cellular uptake by $27.82 \%$ relative to the control. This indicates that clathrin-mediated endocytosis is involved in the transport process of RES-GNPs. Likewise, the cellular uptake of RES-GNPs was greatly inhibited under $4^{\circ} \mathrm{C}$, resulting in reduction of $47.53 \%$. As known, endocytosis is a kind of cytosis transport, in which ATP energy is required. ${ }^{36}$ In addition, the cellular uptake of RES-GNPs strikingly declined under the intervention of galactose, suggesting an uptake

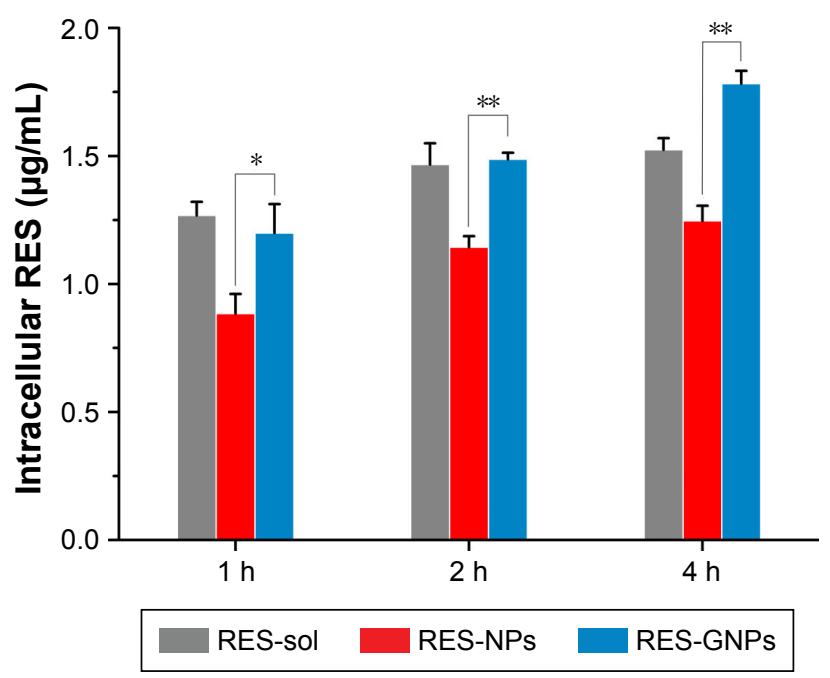

Figure 6 Cellular uptake at I, 2 and $4 \mathrm{~h}$ with a RES level of $20 \mu \mathrm{g} / \mathrm{mL}$ quantified by intracellular RES concentration.

Notes: Paired $t$-test, $* P<0.05$, ${ }^{* * P} P 00.01$, compared with RES-NPs. Data expressed as mean $\pm S D(n=3)$.

Abbreviations: RES, resveratrol; RES-NPs, RES-loaded nanoparticles; RES-GNPs, RES-loaded galactosylated nanoparticles. 


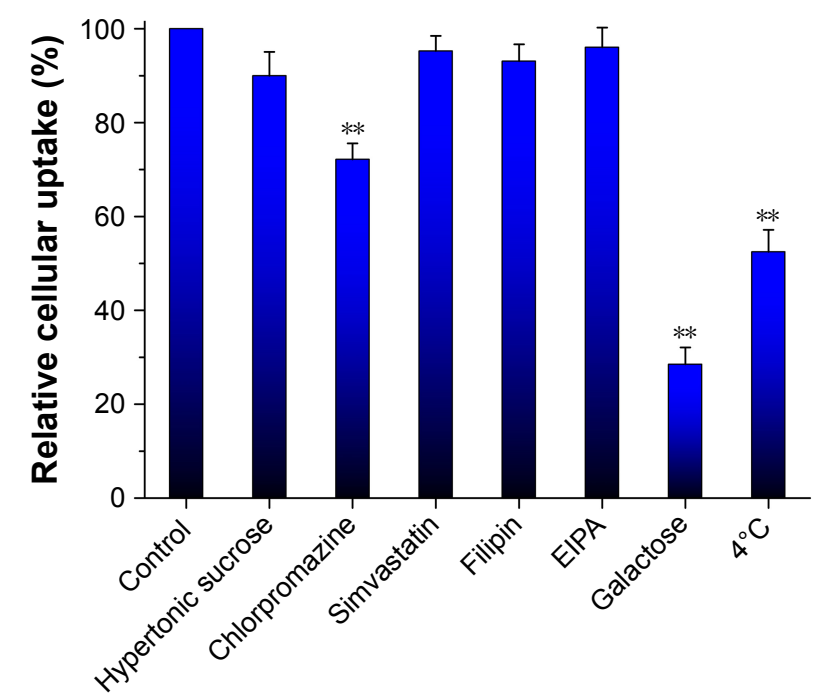

Figure 7 Cellular trafficking mechanisms characterized by relative cellular uptake rate in the presence of physiological inhibitors, galactose or under limited condition. Notes: Paired $t$-test, ${ }^{* *} P<0.01$, compared with control. Data expressed as mean \pm $\mathrm{SD}(\mathrm{n}=3)$.

Abbreviation: EIPA, 3-amino-6-chloro- $\mathrm{N}$-(diaminomethylene)-5-(ethyl(isopropyl) amino)pyrazine-2-carboxamide.

competition between RES-GNPs and galactose. These results indicate that cytosis plays an important role in the cellular trafficking of RES-GNPs, especially the clathrin-mediated endocytosis by ligand-receptor/transporter interaction. ${ }^{37}$

The fluorescent staining of Caco-2 cells treated with coumarin 6-labeled RES-NPs and RES-GNPs is shown in Figure 8. Both RES-NPs and RES-GNPs could be internalized into the cells. NPs (green) colocalized with the cell nucleus (blue), taking on a light green upon overlay. However, at the same incubation time, the intracellular fluorescence intensity treated with RES-GNPs was apparently stronger than that treated with RES-NPs. This demonstrates that RES-GNPs have better cellular internalization and cytosolic delivery ability than RES-NPs.

\section{Improved anti-inflammatory activity}

TNF- $\alpha$ and IL-6 are well-known proinflammatory cytokines, and the level of NO represents the inflammatory degree. They play important roles in the pathological development of many inflammatory diseases. RAW 264.7 macrophages provide a useful platform for the study of anti-inflammatory activity. ${ }^{38,39}$ LPS can conveniently induce upregulation and secretion of proinflammatory cytokines, such as TNF- $\alpha$ and IL-6, and release of NO. In this study, RAW 264.7 macrophages were coincubated with LPS and RES preparations (25 $\mu \mathrm{g} / \mathrm{mL})$. The levels of proinflammatory mediators in different groups are shown in Figure 9. It turned out that RES, RES-NPs and RES-GNPs substantially suppressed expression of TNF- $\alpha$, IL-6 and NO compared to the LPS model. But, there were significant differences in the inhibitory effect among them. RES-GNPs exhibited the highest inhibitory activity on TNF- $\alpha$ release, followed by RES-NPs and then RES. The effects of various RES preparations on IL-6 release were somewhat distinct from TNF- $\alpha$. RES-NPs did not show an advantage over free RES, but RES-GNPs
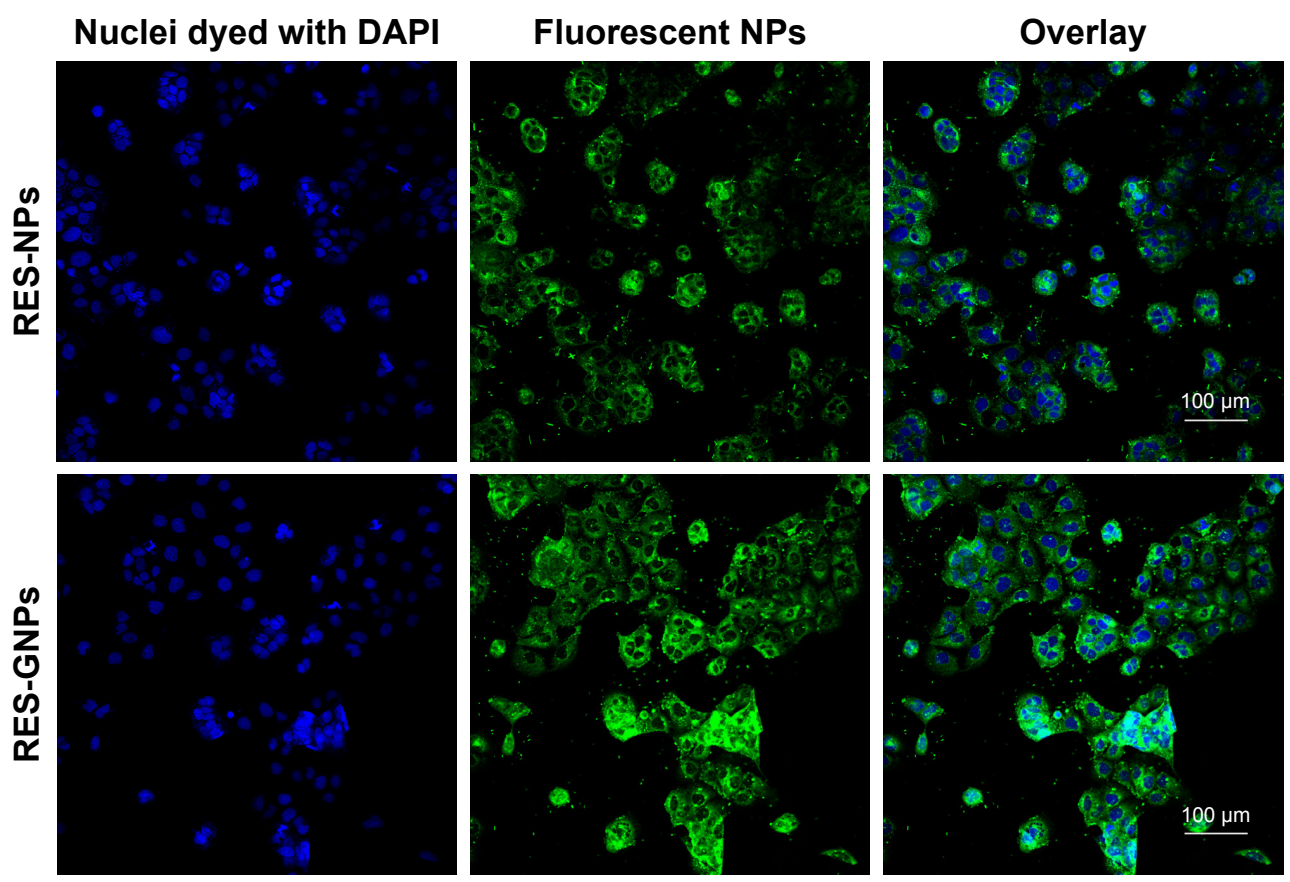

Figure 8 Cellular internalization observed by confocal laser scanning microscopy (scale bar $=100 \mu \mathrm{m}$ )

Abbreviations: RES, resveratrol; RES-NPs, RES-loaded nanoparticles; RES-GNPs, RES-loaded galactosylated nanoparticles. 

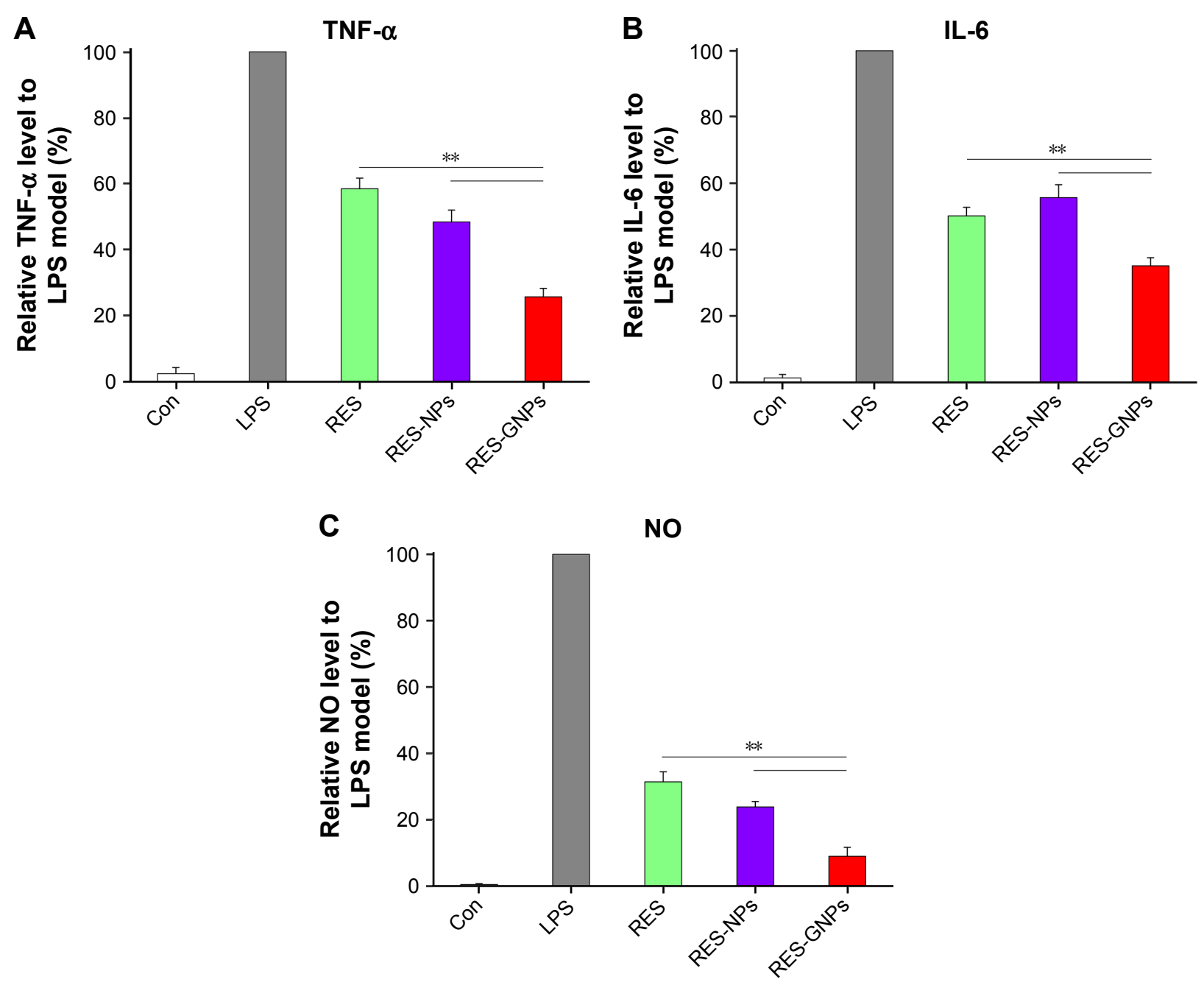

Figure 9 Inhibitory effects of RES, RES-NPs and RES-GNPs on TNF- $\alpha$ (A), IL-6 (B) and NO (C) in LPS-induced RAW264.74 cells. Notes: Data expressed as mean $\pm S D(n=3)$. Paired $t$-test, $* * P<0.01$, compared with RES and RES-NPs.

Abbreviations: TNF, tumor necrosis factor; LPS, lipopolysaccharide; RES, resveratrol; RES-NPs, RES-loaded nanoparticles; RES-GNPs, RES-loaded galactosylated nanoparticles; IL, interleukin; NO, nitric oxide.

gave an unexpected performance on IL-6 inhibition. As for $\mathrm{NO}$, the inhibitory effects of three formulations were more conspicuous, especially for RES-GNPs. RES encapsulated in NPs possess a higher inhibitory activity on NO production compared to free RES. Improvement in anti-inflammation of RES through NPs can be attributed to the enhanced cellular internalization level and prolonged pharmacological action due to sustained drug release.

TNF- $\alpha$ and IL- 6 are representative inflammatory mediators and the level of NO reflects the severity of inflammatory disease. TNF- $\alpha$ is a cell signaling protein (cytokine) involved in the systemic inflammation, which is one of the cytokines that mediate the acute phase reaction. It is mainly produced by activated macrophages, certainly can be generated by many other cell types, such as lymphocytes and dendritic cells. ${ }^{40} \mathrm{IL}-6$ is an interleukin that acts as both a proinflammatory cytokine and an anti-inflammatory cytokine. ${ }^{41} \mathrm{It}$ is secreted by $\mathrm{T}$ cells and macrophages that can stimulate the immune response, thus causing inflammation if overexpressed. NO is a signaling molecule that plays a key role in the pathogenesis of inflammation. It produces an anti-inflammatory effect under normal physiological conditions. Meanwhile, NO is considered as a proinflammatory mediator that induces inflammation due to overproduction in abnormal situations. ${ }^{42}$ Inhibiting production of TNF- $\alpha$, IL- 6 and NO will be of important significance for relieving the inflammatory reaction and controlling the inflammatory progress. Our developed RES-GNPs exhibit an excellent in vitro anti-inflammatory effect, showing a promising application for the treatment of inflammatory diseases, such as myocarditis, nephritis and vasculitis.

\section{Conclusion}

In this study, GNPs were developed and their suitability as oral delivery vehicle of RES was evaluated. It is found that 
our developed RES-GNPs possess excellent formulation performances compared with conventional polymeric NPs. RES-GNPs exhibit a higher intestinal permeability and can significantly enhance the oral bioavailability of RES after administration. Cell uptake and trafficking tests reveal that RES-GNPs can be more easily transported into the enterocytes. The in vitro anti-inflammatory efficacy of RESGNPs was also higher than that of RES-NPs and free RES. Enhanced bioavailability and bioactivity for RES-GNPs is demonstrated to be concerned with galactosylation of NPs that renders them superior transepithelial transport and absorption. The developed formulation not only enhances the oral bioavailability of RES but also strengthen its bioactivity, demonstrating a promising application for inflammatory diseases. This work provides insight into the use of GNPs for oral delivery of poorly water-soluble drugs to potentiate their bioactivity.

\section{Acknowledgments}

This work was supported in part by the National Natural Science Foundation of China (31672563), the National Key Research and Development Program of China (2016YFD0501004) and the Guangdong Provincial Key Laboratory of Prevention and Control for Severe Clinical Animal Diseases (2017B030314142).

\section{Disclosure}

The authors report no conflicts of interest in this work.

\section{References}

1. Xu D, Li Y, Zhang B, et al. Resveratrol alleviate hypoxic pulmonary hypertension via anti-inflammation and anti-oxidant pathways in rats. Int J Med Sci. 2016;13(12):942-954.

2. Andrade Volkart P, Benedetti Gassen R, Muhlen Nogueira B, Nery Porto B, Eduardo Vargas J, Arigony Souto A. Antitumor activity of resveratrol is independent of $\mathrm{Cu}(\mathrm{II})$ complex formation in MCF-7 cell line. Bioorg Med Chem Lett. 2017;27(15):3238-3242.

3. Montesano A, Luzi L, Senesi P, Mazzocchi N, Terruzzi I. Resveratrol promotes myogenesis and hypertrophy in murine myoblasts. $J$ Transl Med. 2013;11:310.

4. Penalva R, Esparza I, Larraneta E, Gonzalez-Navarro CJ, Gamazo C, Irache JM. Zein-based nanoparticles improve the oral bioavailability of resveratrol and its anti-inflammatory effects in a mouse model of endotoxic shock. J Agric Food Chem. 2015;63(23):5603-5611.

5. Yen CC, Chang CW, Hsu MC, Wu YT. Self-nanoemulsifying drug delivery system for resveratrol: enhanced oral bioavailability and reduced physical fatigue in rats. Int J Mol Sci. 2017;18(9):E1853.

6. Amri A, Chaumeil JC, Sfar S, Charrueau C. Administration of resveratrol: What formulation solutions to bioavailability limitations? J Control Release. 2012;158(2):182-193.

7. Walle T. Bioavailability of resveratrol. Ann N Y Acad Sci. 2011;1215: 9-15.

8. Zhou J, Zhou M, Yang FF, et al. Involvement of the inhibition of intestinal glucuronidation in enhancing the oral bioavailability of resveratrol by labrasol containing nanoemulsions. Mol Pharm. 2015;12(4): 1084-1095.
9. Francioso A, Mastromarino P, Masci A, d'Erme M, Mosca L. Chemistry, stability and bioavailability of resveratrol. Med Chem. 2014; 10(3):237-245.

10. Bernkop-Schnurch A. Nanocarrier systems for oral drug delivery: do we really need them? Eur J Pharm Sci. 2013;49(2):272-277.

11. da Luz CM, Boyles MS, Falagan-Lotsch P, et al. Poly-lactic acid nanoparticles (PLA-NP) promote physiological modifications in lung epithelial cells and are internalized by clathrin-coated pits and lipid rafts. J Nanobiotechnology. 2017;15(1):11.

12. Wang J, Li L, Wu L, et al. Development of novel self-assembled ES-PLGA hybrid nanoparticles for improving oral absorption of doxorubicin hydrochloride by P-gp inhibition: in vitro and in vivo evaluation. Eur J Pharm Sci. 2017;99:185-192.

13. Paul PK, Nopparat J, Nuanplub M, Treetong A, Suedee R. Improvement in insulin absorption into gastrointestinal epithelial cells by using molecularly imprinted polymer nanoparticles: microscopic evaluation and ultrastructure. Int J Pharm. 2017;530(1-2):279-290.

14. Shahnaz G, Edagwa BJ, McMillan J, et al. Development of mannoseanchored thiolated amphotericin B nanocarriers for treatment of visceral leishmaniasis. Nanomedicine (Lond). 2017;12(2):99-115.

15. Zhang X, Chen G, Zhang T, Ma Z, Wu B. Effects of PEGylated lipid nanoparticles on the oral absorption of one BCS II drug: a mechanistic investigation. Int J Nanomedicine. 2014;9:5503-5514.

16. Karasov WH. Integrative physiology of transcellular and paracellular intestinal absorption. J Exp Biol. 2017;220(Pt 14):2495-2501.

17. Cura AJ, Carruthers A. Role of monosaccharide transport proteins in carbohydrate assimilation, distribution, metabolism, and homeostasis. Compr Physiol. 2012;2(2):863-914.

18. Mizuma T, Ohta K, Hayashi M, Awazu S. Intestinal active absorption of sugar-conjugated compounds by glucose transport system: implication of improvement of poorly absorbable drugs. Biochem Pharmacol. 1992;43(9):2037-2039.

19. Fachinetti N, Rigon RB, Eloy JO, Sato MR, Dos Santos KC, Chorilli M. Comparative study of glyceryl behenate or polyoxyethylene 40 stearate-based lipid carriers for trans-resveratrol delivery: development, characterization and evaluation of the in vitro tyrosinase inhibition. AAPS PharmSciTech. 2018;19(3):1401-1409.

20. Pujara N, Jambhrunkar S, Wong KY, McGuckin M, Popat A. Enhanced colloidal stability, solubility and rapid dissolution of resveratrol by nanocomplexation with soy protein isolate. $J$ Colloid Interface Sci. 2017;488:303-308.

21. Juere E, Florek J, Bouchoucha M, Jambhrunkar S, Wong KY, Popat A, Kleitz F. In vitro dissolution, cellular membrane permeability, and anti-inflammatory response of resveratrol-encapsulated mesoporous silica nanoparticles. Mol Pharm. 2017;14(12):4431-4441.

22. Mohammadi G, Shakeri A, Fattahi A, Mohammadi P, Mikaeili A, Aliabadi A, Adibkia K. Preparation, physicochemical characterization and anti-fungal evaluation of nystatin-loaded PLGA-glucosamine nanoparticles. Pharm Res. 2017;34(2):301-309.

23. Li W, Zhang T, Ye Y, Zhang X, Wu B. Enhanced bioavailability of tripterine through lipid nanoparticles using broccoli-derived lipids as a carrier material. Int J Pharm. 2015;495(2):948-955.

24. Nozawa T, Imai T. Prediction of human intestinal absorption of the prodrug temocapril by in situ single-pass perfusion using rat intestine with modified hydrolase activity. Drug Metab Dispos. 2011;39(7): 1263-1269.

25. Deng W, Xie Q, Wang H, Ma Z, Wu B, Zhang X. Selenium nanoparticles as versatile carriers for oral delivery of insulin: Insight into the synergic antidiabetic effect and mechanism. Nanomedicine. 2017;13(6): 1965-1974.

26. Ruan BF, Ge WW, Cheng HJ, Xu HJ, Li QS, Liu XH. Resveratrol-based cinnamic ester hybrids: synthesis, characterization, and anti-inflammatory activity. J Enzyme Inhib Med Chem. 2017;32(1):1282-1290.

27. Zhang X, Wang H, Zhang T, Zhou X, Wu B. Exploring the potential of self-assembled mixed micelles in enhancing the stability and oral bioavailability of an acid-labile drug. Eur J Pharm Sci. 2014;62: 301-308. 
28. Balzus B, Sahle FF, Honzke S, et al. Formulation and ex vivo evaluation of polymeric nanoparticles for controlled delivery of corticosteroids to the skin and the corneal epithelium. Eur J Pharm Biopharm. 2017; 115:122-130.

29. Chen Z, Tai Z, Gu F, Hu C, Zhu Q, Gao S. Aptamer-mediated delivery of docetaxel to prostate cancer through polymeric nanoparticles for enhancement of antitumor efficacy. Eur J Pharm Biopharm. 2016; 107:130-141.

30. Marier JF, Vachon P, Gritsas A, Zhang J, Moreau JP, Ducharme MP. Metabolism and disposition of resveratrol in rats: extent of absorption, glucuronidation, and enterohepatic recirculation evidenced by a linkedrat model. J Pharmacol Exp Ther. 2002;302(1):369-373.

31. Pacha J. Development of intestinal transport function in mammals. Physiol Rev. 2000;80(4):1633-1667.

32. Lehmann A, Hornby PJ. Intestinal SGLT1 in metabolic health and disease. Am J Physiol Gastrointest Liver Physiol. 2016;310(11):G887-G898.

33. Zhang J, Tang C, Yin C. Galactosylated trimethyl chitosan-cysteine nanoparticles loaded with Map4k4 siRNA for targeting activated macrophages. Biomaterials. 2013;34(14):3667-3677.

34. Jain A, Kesharwani P, Garg NK, et al. Galactose engineered solid lipid nanoparticles for targeted delivery of doxorubicin. Colloids Surf B Biointerfaces. 2015;134:47-58.

35. Kuhnle G, Spencer JP, Chowrimootoo G, et al. Resveratrol is absorbed in the small intestine as resveratrol glucuronide. Biochem Biophys Res Commun. 2000;272(1):212-217.
36. Selvi RB, Chatterjee S, Jagadeesan D, Chaturbedy P, Suma BS, Eswaramoorthy M, Kundu TK. ATP driven clathrin dependent entry of carbon nanospheres prefer cells with glucose receptors. J Nanobiotechnology. 2012;10:35.

37. Jain A, Jain A, Parajuli P, et al. Recent advances in galactose-engineered nanocarriers for the site-specific delivery of siRNA and anticancer drugs. Drug Discov Today. Epub 2017 Nov 10. doi:10.1016/j.drudis. 2017.11.003.

38. Matsuda Y, Minagawa T, Okui T, Yamazaki K. Resveratrol suppresses the alveolar bone resorption induced by artificial trauma from occlusion in mice. Oral Dis. 2018;24(3):412-421.

39. Xiang FF, He JW, Liu ZX, Peng QZ, Wei H. Two new guaiane-type sesquiterpenes from Curcuma kwangsiensis and their inhibitory activity of nitric oxide production in lipopolysaccharide-stimulated macrophages. Nat Prod Res. Epub 2017 Sep 20:1-6. doi:10.1080/ 14786419.2017.1378203.

40. Fei M, Bhatia S, Oriss TB, et al. TNF-alpha from inflammatory dendritic cells (DCs) regulates lung IL-17A/IL-5 levels and neutrophilia versus eosinophilia during persistent fungal infection. Proc Natl Acad Sci US A. 2011;108(13):5360-5365.

41. Zhang $H$, Chen W. Interleukin 6 inhibition by triptolide prevents inflammation in a mouse model of ulcerative colitis. Exp Ther Med. 2017;14(3): 2271-2276.

42. Sharma JN, Al-Omran A, Parvathy SS. Role of nitric oxide in inflammatory diseases. Inflammopharmacology. 2007;15(6):252-259.
International Journal of Nanomedicine

\section{Publish your work in this journal}

The International Journal of Nanomedicine is an international, peerreviewed journal focusing on the application of nanotechnology in diagnostics, therapeutics, and drug delivery systems throughout the biomedical field. This journal is indexed on PubMed Central, MedLine, CAS, SciSearch $®$, Current Contents ${ }^{\circledR} /$ Clinical Medicine,

\section{Dovepress}

Journal Citation Reports/Science Edition, EMBase, Scopus and the Elsevier Bibliographic databases. The manuscript management system is completely online and includes a very quick and fair peer-review system, which is all easy to use. Visit http://www.dovepress.com/ testimonials.php to read real quotes from published authors. 